\title{
Medication adherence in patients after percutaneous coronary intervention due to acute myocardial infarction: From research to clinical implications
}

\author{
Damian Swieczkowski ${ }^{1}$, Mariusz Mogielnicki ${ }^{2}$, Natalia Cwalina ${ }^{1}$, Grzegorz Zuk ${ }^{1}$, \\ Izabela Pisowodzka ${ }^{1}$, Dariusz Ciecwierz ${ }^{1}$, Marcin Gruchala ${ }^{1}$, Milosz Jaguszewski ${ }^{1}$ \\ ${ }^{1} 1^{\text {st }}$ Department of Cardiology, Medical University of Gdansk, Poland \\ ${ }^{2}$ Research and Development Laboratory, OCEANIC S.A., Poland
}

\begin{abstract}
Adherence to medication is one of the most significant challenges of secondary prevention in patients after an acute myocardial infarction (AMI). Indeed, it has been well established that higher adherence is associated with better cardiovascular outcomes. Research pertaining to secondary prevention after AMI treated with percutaneous coronary intervention (PCI) focuses mainly on the adherence to antiplatelet therapy. Adherence levels have been found to be particularly poor and thus, insufficient with regards to prevention; with a high rate of discontinuation of therapy occurring during the 12-month follow-up. There are numerous predicting factors associated with non-adherence to antiplatelet therapy in patients after PCI. These include, but are not limited to, a lack of education on antiplatelet treatment, various comorbidities, depression, or even, unmarried status. Financial limitations of the patient also play a relevant role, however, the nature of this impediment is problematic and requires further investigation. It would seem beneficial to carry out advanced research based on a randomized and double-blind protocol, however, large-cohort, real-world observations are also essential to investigate nonadherence across a broad array of treatment settings above and beyond the scope of prospective clinical trials. Research about adherence under the context of invasive treatment of AMI has a tremendous practical impact and should be considered a matter of importance concerning both clinicians and scientists. Close collaboration between not only researchers, health practitioners, i.e. physicians and pharmacists, but also politicians, is strongly recommended to aid in designing an intervention that might improve patient adherence. (Cardiol J 2016; 23, 5: 483-490)
\end{abstract}

Key words: medication adherence, compliance, myocardial infarction, acute coronary syndrome, percutaneous coronary intervention, platelet aggregation inhibitors

\section{Introduction}

Adherence to a medication regimen in postmyocardial infarction patients is one of the most significant challenges of secondary prevention in cardiovascular medicine $[1,2]$. Due to the high prevalence of comorbidities, and the multifactorial nature of cardiovascular diseases, the majority of patients often require combination therapy [3]. A complex drug regimen may prove to have a detrimental effect on patient adherence and consequently, play a direct role in poorer therapeutic

Address for correspondence: Damian Świeczkowski, MPharm, $1^{\text {st }}$ Department of Cardiology, Medical University of Gdansk, ul. Dębinki 7, 80-211 Gdańsk, Poland, e-mail: d.swieczkowski@o2.pl

Received: 26.06.2016

Accepted: 14.07.2016 
prognoses [4]. Therefore, improvement of patient adherence should be considered a common goal, not only of healthcare practitioners such as physicians and pharmacists, but also that of the politicians who create both nationwide and international frameworks of healthcare policy [5]. Many different factors associated with dual antiplatelet therapy (DAPT) non-adherence have been investigated in various treatment settings under a broad array of prospective clinical trials. In certain randomized clinical trials (RCTs), high-risk populations such as elderly patients, have been essentially underscored, particularly in acute coronary syndrome (ACS) subsets; this is of capital importance in regards to the interpretation of the diverse results obtained in differently designed studies. In the scientific literature, there is a great deal of advanced research focused on adherence-related matters in regards to acute myocardial infarction (AMI), however, adherence in patients after AMI that have been treated with percutaneous coronary intervention (PCI) still raises many unresolved questions. Patients after AMI with stent implantation are strongly recommended to implement antiplatelet therapy starting with a dual regime, namely DAPT which comprises of acetylsalicylic acid (ASA) and adenosine diphosphate receptor inhibitors e.g. clopidogrel, ticagrelor [6]. The strong correlation between the use of DAPT and the decrease in the rate of mortality after AMI is one of the most accurate and well-documented cause-and-effect relationships in this area of research. As antiplatelet agents are generally widely available, and in many cases, reimbursed by public resources or private insurance policies, it is entirely possible to receive high-quality data from pharmacy refill statistics.

Questions concerning adherence-related issues after invasive treatment of AMI are not only a purely scientific matter, but also bear crucial practical consequences. Defining the important predictors of non-adherence remains a challenge both for clinicians and scientists.

The aim of this paper is to present the current level of knowledge regarding adherence-related issues in the context of AMI treated with PCI. Additionally, we would like to add our voice to the discussion on the practical aspects and implications of research conducted on adherence-related issues.

\section{Treatment adherence: Methodological issues and definition}

Experts, who focus mainly on adherence in their works, state that in their opinion, the current literature available describing adherence-related behaviors is only in the initial stages. They believe this is just the beginning of methodological development [7]. While there are several wellestablished methods to assess adherence, none of them fulfill high-quality scientific and clinical requirements [8]. Moreover, methodological impediments have emerged; for instance, self-reported questionnaires - the cheapest and the easiest way to measure the adherence level, have over- or under-estimated this phenomenon $[9,10]$. It should be noted that medical records, patient databases and pharmacy refill statistics, all which clearly aid in evaluating adherence in a large patient cohort; oversimplify the multidimensional character of adherence [11]. The use of a pill count tracker or electronic bottles may unknowingly change the patients' behavior in regards to medication adherence, and thus, the estimation of adherence provided might prove unreliable. They may also give the impression of continuous supervision of the patient and as a result, influence acceptance of the measurement method and the patients' overall comfort [12].

Incorrect use of adherence-related terms may lead to some confusion and misunderstandings. The differences between adherence and persistence should be clearly explained to the patient and the distinctions emphasized. Patients who are included in the persistent cohort, can also be classified as showing poor adherence, while in the exact same clinical situation. Adherence is a fully qualitative concept, regarding quality use of the medication (Fig. 1). In contrast, persistence should be understood in the quantitative scope $[13,14]$ and might be simply described as the duration of time from initiation to discontinuation of pharmacotherapy (Fig. 1). The concept of adherence is also frequently mistaken with compliance. Both terms might be described as the extent to which patients are consistent with the health practitioner recommendations. At this point, we should just admit, that 'patients' compliance' - frequently employed to describe medication-taking behavior, has a pejorative connotation and, thus, should not be widely used in the scientific deliberation (Fig. 1) [15].

The model of concordance serves a philosophical role, taking into account the shared understanding between patients, physicians, pharmacists, and other healthcare providers. This agreement must be supported by mutual trust and confidence, where the physician and patient serve together as a team, bringing together highly advanced medical knowledge and the patient's care for his or her own health [16]. 


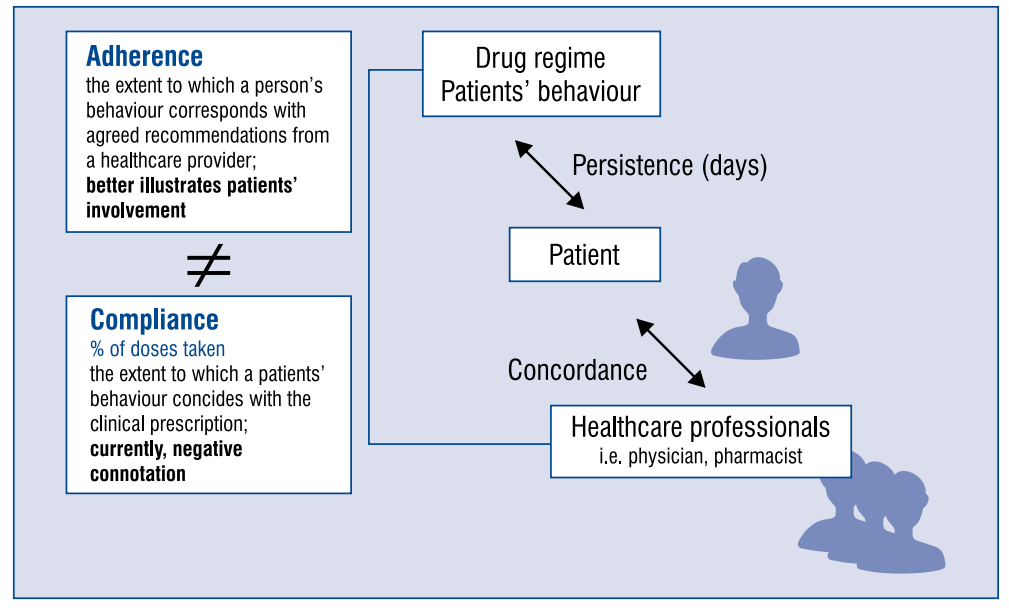

Figure 1. Definitions of adherence, persistence, and compliance.

\section{Adherence after AMI: General assessment}

Non-diagnosed chronic coronary artery disease (CAD) can sometimes initially present as AMI. Due to the wide availability of high-quality medical care, in most cases, the patients' primary condition is already diagnosed and well known. The majority of patients who have suffered an AMI have a long medical history of cardiovascular diseases. This prevalence contributes to maintaining researchers' interest in adherence-related issues in relation to AMI [17]. Patients with high adherence who used statins or beta-blockers were found to have a lower mortality risk when compared to those diagnosed with adherence-related problems [18]. Interestingly enough, long-term observations have indicated that the level of adherence decreases over time, with a 3 -year medication continuation rate of $44 \%$ for statins, $48 \%$ for beta-blockers and $43 \%$ for angiotensin-converting enzyme inhibitors (ACEI)/angiotensin II receptor blockers (ARB) [19]. In France, a 30-month follow-up in patients after AMI estimated the non-adherence rate to be $24 \%$ for statins and $32 \%$ for beta-blockers [20]. Discontinuation of ACEI therapy was discovered at unacceptable rates $-7 \%$ at 1 month following AMI, $22 \%$ at 6 months and approximately $50 \%$ at 1 year. It is possible that financial difficulties were a key factor that could help predict the possibility of discontinuation of the recommended therapy [21].

Every effort to improve adherence should be undertaken in order to optimize pharmacotherapy and achieve better patient outcomes. Nevertheless, as adherence-related problems are multidimensional, $100 \%$-adherence is extremely difficult to achieve, if not impossible. We believe that each case should be assessed individually — allowing us to predict the problems that may occur with our prescribed therapies, anticipate them, and teach our patients how to prevent them. The development of effective interventions has proven to be a tremendous challenge in all areas of medicine [22].

\section{Non-adherence predicting factors: \\ A multidimensional approach}

Adherence is a multidimensional issue, which is why many studies have focused on trying to pinpoint the factors that may constitute a significant contribution to patients' behavior in relation to medication management, especially in the context of AMI (Fig. 2). In one single center observation, both ST-elevation myocardial infarction (vs. non-ST-elevation myocardial infarction) and male gender were suggested to predict non-adherence, while 3-vessel CAD was associated with a higher level of adherence when patients were treated with clopidogrel [23]. Comorbidities such as diabetes, hypertension, or atrial fibrillation may indicate problems not only with compliance regarding the physician's recommendations, but also with turning in the prescription for receipt of antiplatelet drugs $[24,25]$. Elderly patients, smokers, patients with silent ischemia or no previous AMI, or those patients with no coronary artery bypass graft, mainly discontinued DAPT due to non-adherence or bleeding [26]. The patient's state of mental health - e.g. depression [27] or an unmarried status [28] seems to be associated with a higher risk of non-adherence as well. Research has proven, to put it simply, that adherence-related issues depend on the patients' behavior. Patients who have been classified with poor adherence before the initiation of antiplatelet therapy, after AMI may be even 


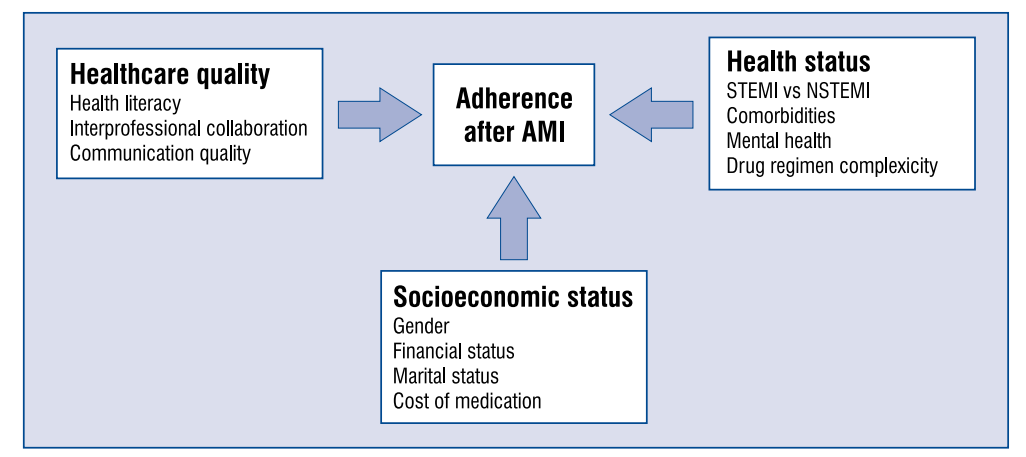

Figure 2. Adherence after acute myocardial infraction (AMI) - summary; STEMI - ST-segment elevation myocardial infarction; NSTEMI — non-ST-segment elevation myocardial infarction.

more likely to discontinue treatment, without any logical reason [29]. As mentioned before, financial limitations may affect adherence as well. Indeed, a lower education level, immigrant status, and poor transitions in care were all documented as common barriers to clopidogrel adherence [30]. Pallares et al. [31] concluded that financial obstacles in the non-adherent vs. adherent group were identified as $42 \%$ vs. $36 \%$. Surprisingly, adherent patients reported a higher co-payment rate [31]. In a different study, $62 \%$ of respondents listed the cost of a medication as a potential reason for their choice to discontinue therapy [28]. Patients already on DAPT, those with previous or in-hospital bleeding, or patients using oral anticoagulation were all determined to have a higher probability of non-adherence, subsequently increasing their risk of stent thrombosis [32]. There is no doubt, that avoiding the side effects of certain medications plays a critical role in a patient's adherence to treatment, especially those with AMI. According to legislation in the European Union, the reporting of adverse drug reactions to the appropriate regulatory authorities is obligatory for physicians, pharmacists and nurses. Other factors proven to play a role in adherence-related issues include poor communication between patients and their physicians, and the patients' lack of knowledge regarding their own medication regimens [33]. Investigating non-adherence within a group of ACS referred for urgent PCI, we must take into account the substantial heterogeneity in baseline characteristics, data sources, and various P2Y12-inhibitor resistance. It is essential to remember the diversity present across a variety of regional medical practices that may play a critical role in the level of adherence amongst patients who were treated differently for ACS. As the side effects and safety profiles of several P2Y12 inhibitors differ, it is hard to address whether rates of adherence vary according to the type of DAPT prescribed [26]. Simplified drug regime, which is directly associated with the improvement of adherence level, led consequently to the introduction of fixed dose combination and polypill concept. Indeed, in patients after AMI, covering a broad range of different socioeconomic strata, the use of polypill strategy increased self-reported and directly-measured medication adherence for secondary prevention [34]. Since number of studies, including RCTs, demonstrated the convenient reduction of multiple risk factors after the reduction of dosage demands, the concept of polypill has been implemented into European Society of Cardiology guidelines [35-37].

\section{Adherence after AMI treated by PCI}

Although new-generation drug-eluting stents (DES) have been designed to decrease device related outcomes, early discontinuation of DAPT is still the most powerful predictor of stent thrombosis. Unfortunately, many patients with ACS frequently discontinue DAPT due to non-adherence or bleeding [26]. DAPT adherence, although high at 1 month, decreases substantially by 6 months [26]. Much of the literature describes the direct relationship of failure to adhere to therapy with poorer patient outcomes [32]. Patients who disrupted their DAPT due to non-adherence or bleeding are at the highest risk of a poorer outcome; those discontinuing DAPT temporarily due to a need for surgery, or permanently due to the completion of therapy, are at a lower risk [32]. Recently published studies have confirmed that adherence in patients after myocardial intervention with PCI could be considered unexpectedly high. In the German population, the adherence level was estimated at $90.8 \%$ for ASA and at $79.2 \%$ for ACEI or ARB. Although the above-mentioned study had a relatively 
large patient cohort, the study was conducted in a single medical center; which could raise some important issues regarding the generalization of results and conclusions [38]. Even a delay in filling the first prescription after invasive coronary treatment could be associated with unexpected, adverse clinical outcomes. Based on data obtained from a British-Columbian consortium, the postponement of handing in a prescription, for more than 3 days projected with possible mortality and/or recurrent AMI [39]. These results are consistent with other studies that analyzed patients who received their prescription for clopidogrel successfully on the day of hospital discharge after DES implantation against patients who delayed the purchase of clopidogrel. The latter group was found to have a higher probability of sudden death or recurrent myocardial infarction $(14.2 \%$ vs. $7.9 \% ; \mathrm{p}<0.001)$ [40]. One study demonstrated that as many as 1 in 7 patients treated with DES discontinued thienopyridines therapy (clopidogrel, prasugrel or ticlopidine) without any reasonable medical reason within the first month following their procedure [41]. French researchers have estimated the risk of discontinuation of therapy after 1 month, in relation to ASA and after 3 months of clopidogrel treatment, at $18.6 \%$, with an increase to $49.1 \%$ after 12 months. It is possible that the adherence-related issues might be a reflection of the well-described phenomena of many patients' resistance to antiplatelet treatment [42]. These results are consistent with the observations of an Italian study where only $54 \%$ of patients during a 6 -month follow-up visit were determined to be receiving appropriate antiplatelet therapy [43]. Zhu et al. [24] estimated the level of adherence for clopidogrel at $66.8 \%$, based on a patient cohort of 10,465 people, aged $18-65$ years.

Although adherence is mainly assessed in relation to clopidogrel, one study has investigated patient adherence to prasugrel, estimating the patient's use of prasugrel with the medication possession ratio (MPR). MPR has proven a suitable tool for the measurement of adherence level in regards to large sample sizes, particularly when data is obtained from pharmacy refill statistics. In the study concerning prasugrel, $69 \%$ of patients were classified within the good adherence group, with an MPR $\geq 80 \%$ [27].

After reviewing the data provided from the 8-item Morisky Medication Adherence Scale (MMAS-8), Muntner et al. [29] observed low adherence in $11 \%$ of patients discharged home after PCI. Harjai et al. [44] in a prospective observation, demonstrated that the continuation of DAPT bey- ond 12 months in patients undergoing PCI of native coronaries, who survived with no major adverse cardiac events (MACE), did not confer long-term protection from death or myocardial infarction. Ferreira-Gonzales et al. [45] has suggested that the discontinuation of DAPT after DES implantation within 1 year following the invasive procedure is not significantly associated with the risk of an occurrence of MACE during the follow-up. In fact, discontinuation, was often of a temporary nature (on average 7 days) and associated with clopidogrel treatment. The level of adherence was estimated based on patients' declarations only, which was a major limitation of the study. A different study reported the increase of MACE due to discontinuation of clopidogrel within the initial 3-month period, but not after 3 to 12 months [46].

\section{Implications on routine practice}

Both physicians in clinical practice and pharmacists should be constantly assessing patient adherence to treatment regimens [47]. Adherencerelated issues are an extremely important subject matter to be discussed during the initial medical examination. In our opinion, a patient's adherence level may drastically change throughout the therapeutic process. During the initial phase of diagnosis and treatment, patients' knowledge about their disease and belief in recovery is much higher than when compared to beliefs after a long period of extensive therapy. This is why physicians should never underestimate adherence-related issues.

Pharmacists can provided adherence-related interventions, based on simple tools such as questionnaires aiming at identifying non-adherent patients [48]. As previously mentioned, one of the latest systematic reviews indicated that a lack of patient education in regards to antiplatelet therapy may have an impact on adherence levels [49]. Indeed, interventions aimed at improving and expanding health education should be established as one of the most promising methods in order to prevent non-adherence. Each healthcare professional has an moral obligation to their patients to not only treat them, but also to educate their patients about their illness and therapies, and thus, should be willing to join in the campaign against non-adherence. Community pharmacies could play an additional role in patient education, as they are easily accessible to patients, have front-line access to refill statistics, and are the medical professional the patient sees most often. Through their dedication to patient adherence activities, pharmacists could help lead to better 
adherence rates among patients in ACS settings undergoing stent implantation. Therefore, the role of a pharmacist should change, from that solely of a medication dispenser, to one that is much more patient-oriented. In countries such as the United Kingdom or Canada - often considered the global capital of advanced pharmaceutical services, these processes are well implemented and have been further introduced into both practical and routine clinical settings [50].

It is crucial that we focus on the improvement of communications with patients - not only in quantity but also, and especially, in quality. Patients desire that physicians and pharmacists speak with them using simple terminology, avoiding unnecessary medical jargon. This focus on quality communications should be undertaken by the healthcare system as a whole, as it plays an important role in patient adherence. After hospitalization, patients should be able to quickly and efficiently familiarize themselves with all the necessary recommendations for further treatment and care, not only concerning pharmacological therapy but also in regards to lifestyle changes, nutrition and addictions. The role of healthcare professionals is to promote adherence-related knowledge in an acceptable, non-intrusive way, allowing the patient to gain control over their own health. It is completely unacceptable for members of healthcare teams to undermine the professional authority of other healthcare practitioners in the presence of a patient. Mutual respect among physicians, nurses, pharmacists and other members of the healthcare system may have a large impact on patient behavior and consequently on their adherence to treatment.

\section{Optimal adherence: A challenge for the near future}

Although patient adherence at times seems an uncertain guessing game, the development of evidence-based interventions are strongly recommended, particularly actions that help promote adherence [51]. The necessity to conduct RCTs has been frequently pointed out in relevant literature, especially in the context of distinguishing interventions aimed at primary and secondary prevention of cardiovascular diseases [52]. Patients suffering from cardiovascular diseases, who received assistance from their pharmacist, exhibited a decrease in the systolic and diastolic blood pressure, total cholesterol or - in the context of public health issues - showed a decrease in smoking habits [53]. Of note, one single-center observation showed that telephone contact with patients who were implan- ted with DES resulted in "near-perfect" DAPT adherence. Phone calls were made by nurses 7 days after the procedure, and then 1,6 , and 9 months following hospital discharge. The protocol of the trial was based on randomized selection. Whether these results could be implemented broadly, particularly in patients with ACS, is worthy of further investigation [54].

From the methodological point of view, research should focus on pinpointing the connections between the different ways of measuring adherence. As most antiplatelet drugs are reimbursed, the comparison of data obtained from pharmacy refills and from self-reported questionnaires, is not so complicated. To fully understand adherencerelated issues, research should not only focus on adherence to antiplatelet therapy but other therapies as well. The limited number of works estimating adherence related to different types of medication other than antiplatelet agents could lead to an underestimation of the problem.

Moreover, in our opinion, the clinical 'resistance' to antiplatelet treatment that is described in the literature may be in fact, associated with poor adherence [55, 56]. Research could help distinguish the reasons for this phenomena - those which are due to poor adherence, in contrast with those which might be associated with an unexplored pharmacological background.

Last but not least, the strong need for large-cohort and multi-center studies in regards to patient adherence should be emphasized. As such, followup monitoring should be extended to a longer time period -5 years or more, which we believe crucial from an epidemiological point of view.

To summarize, the first and most essential change to improve adherence, is to expand the communication between patients and physicians, both in quality and quantity. This is an extremely important target for interventions aimed at increasing DAPT adherence. Implementing a larger role of clinical pharmacists on the cardiology wards, and in follow-up care, could potentially improve adherence in patients undergoing PCI after ACS. Interprofessional collaboration is strongly needed to help realize and execute direct patient education. Indeed, interventions aimed at improving and expanding health education should be established as one of the most promising methods in order to prevent non-adherence.

Adherence-related issues require more profound and advanced research, especially in the context of AMI treated with PCI. Currently, the majority of studies are conducted with regards to 
antiplatelet therapy [57]. Assessment of adherence to different types of medication or those estimating the adherence to non-pharmacological treatment e.g. lifestyle recommendations, in large-cohorts is nowadays strongly desired. The development of effective interventions to improve adherence has proven to be a tremendous challenge in all areas of medicine; and as such, requires a multi-disciplinary focus, as adherence is an issue that concerns both healthcare practitioners and scientists.

This paper contributes to the global discussion on the incremental implications of research in adherence-related issues.

\section{Conflict of interest: None declared}

\section{References}

1. Choudhry NK, Glynn RJ, Avorn J et al. Untangling the relationship between medication adherence and post-myocardial infarction outcomes: Medication adherence and clinical outcomes. Am Heart J, 2014; 167: 51-58.e5. doi: 10.1016/j.ahj.2013.09.014.

2. Kuepper-Nybelen J, Hellmich M, Abbas S, Ihle P, Griebenow R, Schubert I. Association of long-term adherence to evidencebased combination drug therapy after acute myocardial infarction with all-cause mortality. A prospective cohort study based on claims data. Eur J Clin Pharmacol, 2012; 68: 1451-1460. doi: 10.1007/s00228-012-1274-x.

3. Gierlotka M, Zdrojewski T, Wojtyniak B et al. Incidence, treatment, in-hospital mortality and one-year outcomes of acute myocardial infarction in Poland in 2009-2012: Nationwide AMI-PL database. Kardiol Pol, 2015; 73: 142-158. doi: 10.5603/ KP.a2014.0213.

4. DiMatteo MR, Giordani PJ, Lepper HS, Croghan TW. Patient adherence and medical treatment outcomes: A metaanalysis. Med Care, 2002; 40: 794-811. doi: 10.1097/01. MLR.0000024612.61915.2D.

5. Kolandaivelu K, Leiden BB, O'Gara PT, Bhatt DL. Non-adherence to cardiovascular medications. Eur Heart J, 2014; 35: 3267-3276. doi: 10.1093/eurheartj/ehu364.

6. Grines CL, Bonow RO, Casey DE et al. Prevention of premature discontinuation of dual antiplatelet therapy in patients with coronary artery stents: A science advisory from the American Heart Association, American College of Cardiology, Society for Cardiovascular Angiography and Interventions. J Am Coll Cardiol, 2007; 49: 734-739. doi: 10.1016/j.jacc.2007.01.003.

7. Aslani P, Schneider MP. Adherence: The journey of medication taking, are we there yet? Int J Clin Pharm, 2014; 36: 1-3. doi: 10.1007/s11096-013-9901-x.

8. Lehmann A, Aslani P, Ahmed R et al. Assessing medication adherence: Options to consider. Int J Clin Pharm,2014; 36: 55-69. doi: 10.1007/s11096-013-9865-x.

9. Delamatera M. Improving Patient Adherence. Clin Diabetes, 2006; 24: 71-77. doi: 10.2337/diaclin.24.2.71.

10. Lavsa SM, Holzworth A, Ansani NT. Selection of a validated scale for measuring medication adherence. J Am Pharm Assoc, 2011; 51: 90-94. doi: 10.1331/JAPhA.2011.09154.

11. Van Dalem J, Krass I, Aslani P. Interventions promoting adherence to cardiovascular medicines. Int J Clin Pharm, 2012; 34: 295-311. doi: 10.1007/s11096-012-9607-5.
12. Ye S, Krupka DJ, Davidson KW. Diagnosing medication nonadherence in a patient with myocardial infarction. Front Psychol, 2012; 3: 267. doi: 10.3389/fpsyg.2012.00267.

13. Osterberg L, Blaschke T. Adherence to medication. N Engl J Med, 2005; 353: 487-497. doi: 10.1056/NEJMra050100.

14. Gardner CL. Adherence: A concept analysis. Int J Nurs Knowl, 2015; 26: 96-101. doi: 10.1111/2047-3095.12046.

15. Zeber JE, Manias E, Williams AF et al. A systematic literature review of psychosocial and behavioral factors associated with initial medication adherence: A report of the ISPOR medication adherence and persistence special interest group. Value Health, 2013; 16: 891-900. doi: 10.1016/j.jval.2013.04.014.

16. Bell JS, Airaksinen MS, Lyles A, Chen TF, Aslani P. Concordance is not synonymous with compliance or adherence. $\mathrm{Br}$ J Clin Pharmacol, 2007; 64: 710-711. doi: 10.1111/j.13652125.2007.02971_1.x.

17. Ho PM, Bryson CL, Rumsfeld JS. Medication adherence: Its importance in cardiovascular outcomes. Circulation, 2009; 119: 3028-3035. doi: 10.1161/CIRCULATIONAHA.108.768986.

18. Rasmussen JN, Chong A, Alter DA. Relationship between adherence to evidence-based pharmacotherapy and long-term mortality after acute myocardial infarction. JAMA, 2007; 297: 177-186. doi: 10.1001/jama.297.2.177.

19. Shah ND, Dunlay SM, Ting HH et al. Long-term medication adherence after myocardial infarction: Experience of a community. Am J Med, 2009; 122: 961.e7-e13. doi: 10.1016/j.amjmed.2008.12.021.

20. Tuppin P, Neumann A, Danchin N et al. Evidence-based pharmacotherapy after myocardial infarction in France: Adherenceassociated factors and relationship with 30-month mortality and rehospitalization. Arch Cardiovasc Dis, 2010; 103: 363-375. doi: 10.1016/j.acvd.2010.05.003.

21. Akincigil A, Bowblis JR, Levin C, Jan S, Patel M, Crystal S. Long-term adherence to evidence based secondary prevention therapies after acute myocardial infarction. J Gen Intern Med, 2008; 23: 115-121. doi: 10.1007/s11606-007-0351-9.

22. Choudhry NK, Winkelmayer WC. Medication adherence after myocardial infarction: A long way left to go. J Gen Intern Med, 2008; 23: 216-218. doi: 10.1007/s11606-007-0478-8.

23. Kubica A, Kasprzak M, Obońska K et al. Discrepancies in assessment of adherence to antiplatelet treatment after myocardial infarction. Pharmacology, 2015; 95: 50-58. doi: 10.1159/000371392.

24. Zhu B, Zhao Z, McCollam P et al. Factors associated with clopidogrel use, adherence, and persistence in patients with acute coronary syndromes undergoing percutaneous coronary intervention. Curr Med Res Opin, 2011; 27: 633-641. doi: 10.1185/03007995.2010.551657.

25. Cuisset T, Quilici J, Fugon L et al. Non-adherence to aspirin in patients undergoing coronary stenting: Negative impact of comorbid conditions and implications for clinical management. Arch Cardiovasc Dis, 2011; 104: 306-312. doi: 10.1016/j.acvd.2011.03.091.

26. Czarny MJ, Nathan AS, Yeh RW et al. Adherence to dual antiplatelet therapy after coronary stenting: A systematic review. Clic Cardiol, 2014; 37: 505-513. doi: 10.1002/clc.22289.

27. Nordstrom BL, Simeone JC, Zhao Z et al. Adherence and persistence with prasugrel following acute coronary syndrome with percutaneous coronary intervention. Am J Cardiovasc Drugs, 2013; 13: 263-271. doi: 10.1007/s40256-013-0028-1.

28. Quadros AS, Welter DI, Camozzatto FO et al. Identifying patients at risk for premature discontinuation of thienopyridine after coronary stent implantation. Am J Cardiol, 2011; 107: 685-689. doi: 10.1016/j.amjcard.2010.10.045. 
29. Muntner P, Mann DM, Woodward M et al. Predictors of low clopidogrel adherence following percutaneous coronary intervention. Am J Cardiol, 2011; 108: 822-827. doi: 10.1016/j.amjcard.2011.04.034.

30. Decker C, Garavalia L, Garavalia B, Spartus JA. Clopidogrel-taking behavior by drug-eluting stent patients: Discontinuers versus continuers. Patient Prefer Adherence, 2008; 2: 167-175.

31. Pallares MJ, Powers ER,Zwerner PL, Fowler A, Reeves R, NappiJM. Barriers to clopidogrel adherence following placement of drug-eluting stents. Ann Pharmacother, 2009; 43: 259-267. doi: 10.1345/aph.1L286.

32. Mehran R, Baber U, Steg PG et al. Cessation of dual antiplatelet treatment and cardiac events after percutaneous coronary intervention (PARIS): 2 year results from a prospective observational study. Lancet, 2013; 382: 1714-1722. doi: 10.1016/ S0140-6736(13)61720-1.

33. Garavalia L, Ho PM, Garavalia B et al. Clinician-patient discord: Exploring differences in perspectives for discontinuing clopidogrel. Eur J Cardiovasc Nurs, 2011; 10: 50-55. doi: 10.1016/j. ejcnurse.2010.04.002.

34. Castellano JM, Sanz G, Penalvo JL et al. A polypill strategy to improve adherence: Results from the FOCUS project. J Am Coll Cardiol, 2014; 64: 2071-2082. doi: 10.1016/j.jacc.2014.08.021.

35. Lawrence G, Weintraub KM, Narayan V et al. Combination pharmacotherapy for cardiovascular disease. Ann Intern Med, 2005; 143: 593-599. doi: 10.7326/0003-4819-143-8-200510180-00010.

36. Yusuf S, Pais P, Afzal R et al. Effects of a polypill (Polycap) on risk factors in middle-aged individuals without cardiovascular disease (TIPS): A phase II, double-blind, randomised trial. Lancet, 2009; 373: 1341-1351. doi: 10.1016/S0140-6736(09)60611-5.

37. Perk J, De Backer G, Gohlke H et al. European Guidelines on cardiovascular disease prevention in clinical practice (version 2012). The Fifth Joint Task Force of the European Society of Cardiology and Other Societies on Cardiovascular Disease Prevention in Clinical Practice (constituted by representatives of nine societies and by invited experts). Eur Heart J, 2012; 33: 1635-1701. doi: 10.1093/eurheartj/ehs092.

38. Reuter H, Markhof A, Scholz S et al. Long-term medication adherence in patients with ST-elevation myocardial infarction and primary percutaneous coronary intervention. Eur J Prev Cardiol, 2015; 22: 890-898. doi: 10.1177/2047487314540385.

39. Cruden NL, Din JN, Janssen C et al. Delay in filling first clopidogrel prescription after coronary stenting is associated with an increased risk of death and myocardial infarction. J Am Heart Assoc, 2014; 3: e000669. doi: 10.1161/JAHA.113.000669.

40. Ho PM, Tsai TT, Maddox TM et al. Delays in filling clopidogrel prescription after hospital discharge and adverse outcomes after drug-eluting stent implantation: Implications for transitions of care. Circ Cardiovasc Qual Outcomes, 2010; 3: 261-266. doi: 10.1161/CIRCOUTCOMES.109.902031.

41. Spertus JA, Kettelkamp R, Vance C et al. Prevalence, predictors, and outcomes of premature discontinuation of thienopyridine therapy after drug-eluting stent placement: Results from the PREMIER registry. Circulation, 2006; 113: 2803-2809. doi: 10.1161/CIRCULATIONAHA.106.618066.

42. Latry P, Martin-Latry K, Lafitte M, Peter C, Couffinhal T. Dual antiplatelet therapy after myocardial infarction and percutaneous coronary intervention: Analysis of patient adherence using a French health insurance reimbursement database. EuroIntervention, 2012; 7: 1413-1419. doi: 10.4244/EIJV7I12A221.
43. Pinnarelli L, Mayer F, Bauleo L et al. Adherence to antiplatelet therapy after percutaneous coronary intervention: A population study in a region of Italy. J Cardiovasc Med (Hagerstown), 2015; 16: 230-237. doi: 10.2459/JCM.0000000000000070.

44. Harjai KJ, Shenoy C, Orshaw P, Boura J. Dual antiplatelet therapy for more than 12 months after percutaneous coronary intervention: Insights from the Guthrie PCI Registry. Heart, 2009; 95: 1579-1586. doi: 10.1136/hrt.2009.169896.

45. Ferreira-González I, Marsal JR, Ribera A et al. Double antiplatelet therapy after drug-eluting stent implantation: Risk associated with discontinuation within the first year. J Am Coll Cardiol, 2012; 60: 1333-1339. doi: 10.1016/j.jacc.2012.04.057.

46. Thim T, Johansen MB, Chisholm GE et al. Clopidogrel discontinuation within the first year after coronary drug-eluting stent implantation: An observational study. BMC Cardiovasc Disord, 2014; 14: 100. doi: 10.1186/1471-2261-14-100.

47. Desai NR, Choudhry NK. Impediments to adherence to post myocardial infarction medications. Curr Cardiol Rep, 2013; 15: 322. doi: 10.1007/s11886-012-0322-6.

48. Mc Namara KP, George J, O’Reilly SL et al. Engaging community pharmacists in the primary prevention of cardiovascular disease: Protocol for the Pharmacist Assessment of Adherence, Risk and Treatment in Cardiovascular Disease (PAART CVD) pilot study. BMC Health Serv Res, 2010; 10: 264. doi: 10.1186/1472-6963-10-264.

49. Czarny MJ, Nathan AS, Yeh RW, Mauri L. Adherence to dual antiplatelet therapy after coronary stenting: A systematic review. Clin Cardiol, 2014; 37: 505-513. doi: 10.1002/clc.22289.

50. Merks P, Kaźmierczak J, Olszewska AE et al. Comparison of factors influencing patient choice of community pharmacy in Poland and in the UK, and identification of components of pharmaceutical care. Patient Prefer Adherence, 2014; 14: 715-726. doi: 10.2147/PPA.S53829.

51. Eisenstein EL, Wojdyla D, Anstrom KJ et al. Evaluating the impact of public health notification: Duke clopidogrel experience. Circ Cardiovasc Qual Outcomes, 2012; 5: 767-774. doi: 10.1161/ CIRCOUTCOMES.111.963330.

52. Jalal ZS, Smith F, Taylor D, Patel H, Finlay K, Antoniou S. Pharmacy care and adherence to primary and secondary prevention cardiovascular medication: A systematic review of studies. Eur J Hosp Pharm Sci Pract, 2014; 21: 238-244. doi: 10.1136/ejhpharm-2014-000455.

53. Santschi V, Chiolero A, Burnand B, Colosimo AL, Paradis G. Impact of pharmacist care in the management of cardiovascular disease risk factors a systematic review and meta-analysis of randomized trials. Arch Intern Med, 2011; 171: 1441-1453.

54. Rinfret S, Rodés-Cabau J, Bagur R et al. Telephone contact to improve adherence to dual antiplatelet therapy after drug-eluting stent implantation. Heart, 2013; 99: 562-569. doi: 10.1136/ heartjnl-2012-303004.

55. Kolandaivelu K, Bhatt DL. Overcoming "resistance" to antiplatelet therapy: targeting the issue of nonadherence. Nat Rev Cardiol, 2010; 7: 461-467. doi: 10.1038/nrcardio.2010.71.

56. Postuła M, Tarchalska-Kryńska B, Filipiak KJ et al. Factors responsible for "aspirin resistance" — can we identify them? Kardiol Pol, 2010; 68: 403-411.

57. Koziński M, Obońska K, Stankowska K et al. Prasugrel overcomes high on-clopidogrel platelet reactivity in the acute chase of acute coronary syndrome and maintains its antiplatelet potency at 30-day follow-up. Cardiol J, 2014; 21: 547-556. doi: 10.5603/CJ.a2014.0026. 